\title{
Strategies for the Inclusion of Human Members within Human-Robot Teams
}

\author{
Sarah Strohkorb Sebo, Ling Liang Dong, Nicholas Chang, and Brian Scassellati \\ [sarah.sebo,ling.dong,nicholas.chang,brian.scassellati]@yale.edu \\ Yale University \\ New Haven, CT
}

\begin{abstract}
Team member inclusion is vital in collaborative teams. In this work, we explore two strategies to increase the inclusion of human team members in a human-robot team: 1) giving a person in the group a specialized role (the 'robot liaison') and 2) having the robot verbally support human team members. In a human subjects experiment ( $N=26$ teams, 78 participants), groups of three participants completed two rounds of a collaborative task. In round one, two participants (ingroup) completed a task with a robot in one room, and one participant (outgroup) completed the same task with a robot in a different room. In round two, all three participants and one robot completed a second task in the same room, where one participant was designated as the robot liaison. During round two, the robot verbally supported each participant 6 times on average. Results show that participants with the robot liaison role had a lower perceived group inclusion than the other group members Additionally, when outgroup members were the robot liaison, the group was less likely to incorporate their ideas into the group's final decision. In response to the robot's supportive utterances, outgroup members, and not ingroup members, showed an increase in the proportion of time they spent talking to the group. Our results suggest that specialized roles may hinder human team member inclusion, whereas supportive robot utterances show promise in encouraging contributions from individuals who feel excluded.
\end{abstract}

\section{CCS CONCEPTS}

- Human-centered computing $\rightarrow$ User studies; Empirical studies in collaborative and social computing.

\section{KEYWORDS}

Human-Robot Interaction, Inclusion, Groups and Teams

\section{ACM Reference Format:}

Sarah Strohkorb Sebo, Ling Liang Dong, Nicholas Chang, and Brian Scassellati. 2020. Strategies for the Inclusion of Human Members within HumanRobot Teams. In Proceedings of the 2020 ACM/IEEE International Conference on Human-Robot Interaction (HRI '20), March 23-26, 2020, Cambridge, United Kingdom. ACM, New York, NY, USA, 9 pages. https://doi.org/10.1145/ 3319502.3374808

Permission to make digital or hard copies of all or part of this work for personal or classroom use is granted without fee provided that copies are not made or distributed for profit or commercial advantage and that copies bear this notice and the full citation on the first page. Copyrights for components of this work owned by others than the author(s) must be honored. Abstracting with credit is permitted. To copy otherwise, or republish, to post on servers or to redistribute to lists, requires prior specific permission and/or a fee. Request permissions from permissions@acm.org.

HRI '20, March 23-26, 2020, Cambridge, United Kingdom

(c) 2020 Copyright held by the owner/author(s). Publication rights licensed to ACM.

ACM ISBN 978-1-4503-6746-2/20/03 . \$ $\$ 15.00$

https://doi.org/10.1145/3319502.3374808

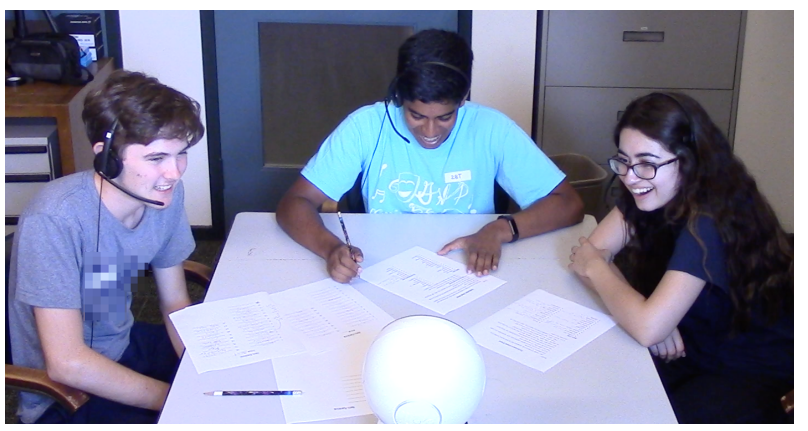

Figure 1: Three participants completed a collaborative task with a Jibo robot, where the robot used two distinct strategies to enhance the inclusion of the human team members.

\section{INTRODUCTION}

Inclusion is increasingly being recognized as an essential component to productive and successful groups and teams [24]. Inclusive work teams are comprised of members, with diverse perspectives and skills, who are well trained and given the opportunity to contribute equally in a group [22]. An individual's inclusion within a group meets both their needs for uniqueness as well as belongingness [29]. Work teams that have an inclusive environment have been demonstrated to produce committed team members and betterperforming teams [5, 27, 29].

As social robots become members of work teams consisting of both humans and robots, it is important to consider the possible influence of the robot on the inclusion of their human team members. Prior work within the field of human-robot interaction (HRI) has shown that robots are capable of shaping group dynamics (e.g. group cohesion [30]) and related behaviors (e.g. conflict management [14, 28], balanced participation [36], and vulnerable expression [32]). Therefore, it is reasonable to assume that the actions of a robot could both positively and negatively influence their human team members' perceived inclusion. Additionally, if a robot is able to increase the inclusion of their human team members, the team is likely to benefit in both the commitment of their team members and in the team's overall performance.

In this work, we are interested in investigating two strategies for enhancing the inclusion of human team members through interactions with a robot in a collaborative task setting. The first strategy we investigate is giving a member of the team a specialized role, where only that team member can ask the robot questions related to the task. Our exploration of a specialized role to interface with a robot is especially relevant to the HRI community because robots are commonly incorporated into human-robot teams by training 
one person to operate the robot (e.g. factory teams, search and rescue teams, surgical teams). The second strategy that we explore is having the robot give verbal support to human team members, such as, "Luis, I think that's worth considering." We evaluate the efficacy of these two strategies in a human subjects experiment where three people and a robot complete a collaborative task. One of the human team members is given the 'robot liaison' role, being the only one who can ask the robot questions to gather more task-related information, and all team members receive verbal support from the robot. We assess the influence of these two inclusion strategies on human team member inclusion by analyzing participants' perceived inclusion ratings, conversational dynamics, and task decisions made by the group.

\section{BACKGROUND}

We situate our work within existing HRI research that has demonstrated a robot's ability to shape group dynamics and affect. We also review literature that speaks to the possible efficacy of two strategies of inclusion we investigate in our experimental study.

\subsection{Robots Interacting with Human Groups}

In prior HRI work, robots have demonstrated their ability to influence the dynamics and behavior of human groups. Direct forms of robot influence in collaborative group tasks, such as moderation of game play and asking directed questions, have been shown to shape human perceptions of group cohesion [30] and the team's performance [31]. In response to conflict between human members of a team, robots have been shown to both heighten the team's awareness of a conflict [14] and, in a setting with children, successfully mediate the conflict [28]. A robot's vulnerable behavior was shown to have 'ripple effects' in a human-robot group, where the human members of the group were more likely to exhibit vulnerable behavior if the robot had also done so [32]. Lastly, a microphone robot that oriented itself to encourage verbal participation from quiet members of a group, displayed success in increasing the evenness of conversational backchanneling between team members [36].

In addition to influencing the dynamics and behavior of group members, robots have also been shown to shape affect experienced and expressed within groups. A robot that expressed empathy toward one player in a game was rated as having characteristics descriptive of a friend [21, 25]. In a game with two human players and two robot players, the human partners directed their gaze more often to a relationship-driven robot when they were partners with it and to a competitive robot when they were opponents with it [23] In a similar setup with two human and two robot players, people displayed higher levels of affinity, group identification, and group trust toward a robot that expressed group-based emotions than toward a robot that expressed individual-based emotions [7]. Robot interventions in a variety of settings have also led to positive results of affect: a robot therapist demonstrated improvements in couples' intimacy and positive affect [37]; a robot used as a therapy assistive tool for pediatric oncology patients was shown to relieve stress, depression, and anger in children [1]; and a robot programmed to guide the elderly in a walking group positively influenced the group's coherence and motivation [10]. Despite the growing body of work examining a robot's capability to shape group affect, to our knowledge no work has yet investigated how robots can influence the inclusion of a human team member in a human-robot group.

\subsection{Strategies to Influence Inclusion}

We review related work that investigates inclusion within groups as well as literature that highlights the potential efficacy of two strategies of including human team members: giving a member a specialized role to interact with the robot and supporting human team members with targeted utterances from the robot.

2.2.1 Inclusion within Groups. Inclusion within human groups has been researched extensively in psychology, as it relates to the formation of subgroups, otherwise known as intergroup biases. Tajfel describes group identification in terms of two necessary components: a cognitive awareness of membership in that group and an evaluation of the value of that membership [35]. This awareness of intergroup membership results in an "us vs. them," or ingroup vs. outgroup, mentality, resulting in behaviors that further reinforce discriminatory ingroup and outgroup relationships [2]. Dunham et al. show that "mere membership" in randomly selected groups result in ingroup-favoring and outgroup-opposing behaviors in people even as young as 5 years old [8].

2.2.2 Strategy 1: Specialized Roles in Groups. If given a specialized role to interact with a robot in the context of a human-robot team, it is possible that the role might give a sense of value to the team member, enabling them to contribute uniquely to the group. However, it is also possible that the role might further isolate them from the group. A person's perception of their isolation or inclusion within a group is often determined by the existence and perception of ingroups and outgroups and where one stands in relation to the rest of the members. Faultlines, divisions in a group along a salient characteristic (e.g. age, gender), may determine how these ingroup and outgroup relationships are formed [19]. A faultline could be created by giving a member a leadership role or another similarly specialized role. The research on the relationship between leadership and isolation suggests that giving a member a specialized role in the group may lead to feelings of exclusion [26]. The relationship between inclusion and specialized roles in human teams has been studied extensively; however, to our knowledge, no research has been conducted on how specialized roles impact perceptions of inclusion in human-robot teams.

Research Question 1: How does being given a specialized role to interact with a robot influence a human team member's inclusion in a human-robot team?

2.2.3 Strategy 2: Verbal Support. Teams with high levels of inclusion consist of members who "feel their values and norms are supported" [5]. Support within teams may encompass encouraging ideas, acknowledging accomplishments, providing assistance, or simply backchanneling [11]. Backchanneling, one form of verbal support that has been researched extensively, has been defined as "the short utterances produced by one participant in a conversation while the other is talking" [38]. Many consider backchanneling to include nonverbal signals as well, including nodding, facial expressions, and directional gaze [33]. All feedback responses, regardless of form, serve the same function of confirming that the "speaker 

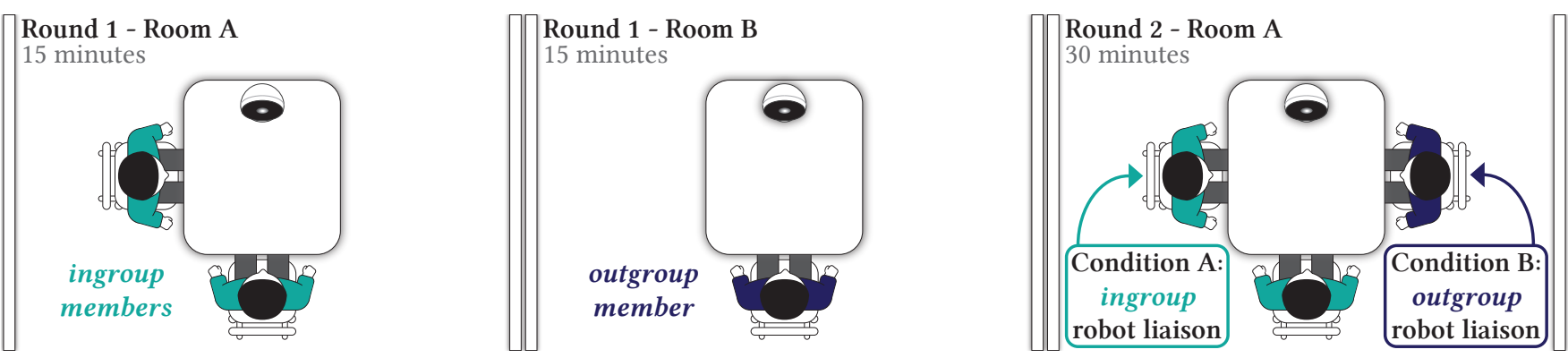

Figure 2: In round 1 of the experiment, two participants (ingroup) and a Jibo robot completed a task in room A while one participant (outgroup) and a Jibo robot completed the same task in room $B$. The outgroup participant joined the two ingroup participants and the robot in room A for round 2 of the experiment, where one of the members is designated the robot liaison.

and listener share a common frame of reference" without threatening the speaker's position as primary speaker [33]. And though responsive feedback may not always be positive or supportive, research has shown that unsupportive verbal feedback does not occur frequently [33]. Given the engaging and communicative nature of backchanneling as well as the importance of team members support, we have reason to believe that supportive utterances from a robot may influence the inclusion of human team members. Although work within HRI has demonstrated the efficacy of robot backchanneling to communicate that the robot is attentively listening [20], no work to our knowledge has investigated the influence of supportive utterances on human inclusion within groups.

Research Question 2: How do supportive utterances from a robot influence a human team member's inclusion in a human-robot team?

\section{METHODS}

In this section, we describe a human subjects experiment investigating the influence of a specialized role involving interaction with a robot and the influence of supportive utterances on the inclusion of human members within a human-robot team.

\subsection{Experiment Design}

We designed a between-subjects experiment where three human participants and a robot work together on a collaborative task. To study the influence of the robot on participants who may experience exclusion, we attempted to artificially form an ingroup and outgroup within the three participants. We did this by having participants first complete round one of the task independently within assigned subgroups of sizes one (the outgroup member) and two (the ingroup members), and then gathered them as a team to complete a second round, see Figure 2. During round two, we studied the influence of a specialized role by assigning one of the three human participants as the robot liaison, the sole human member with the ability to ask the robot information. These designations of human participants led to our two between-subjects conditions:

- Condition A: the robot liaison is an ingroup member

- Condition B: the robot liaison is an outgroup member

Using this experimental design, we addressed our two research questions described in Section 2. For our first research question, we investigated the influence of a specialized role to interact with a robot by examining the difference in inclusion and related behaviors of both ingroup and outgroup participants with the robot liaison role. For our second research question, regardless of condition and robot liaison designation, the robot targeted each participant in the group with supportive utterances (Section 3.3). We are able to measure each participant's reactions to these supportive utterances from the robot and other measures of inclusion, and investigate if the ingroup-outgroup or robot liaison designations influence the effect of the robot's supportive utterances.

\subsection{Collaborative Task: The Survival Problem}

For this experiment, we designed a collaborative task where we asked players to assign ranks to items from a given list based on how useful each item would be for survival in a hostile environment. This task is a derivative of the Desert Survival Problem [18], a commonly used task in HRI groups research [4, 17, 36].

In the first round of the task, players were given 15 minutes to construct an ordered list of items, ranked by importance for survival, from a list of 25 common household items (e.g. umbrella, whistle, watch). During this round, players interacted with a social robot through verbal queries about the time remaining in the round and to learn more information about the survival items. For example, when queried about the survival item 'soda,' the robot responded with " 6 aluminum cans of Coca-Cola. The cans are held in cardboard and the whole pack is wrapped in plastic."

In the second round of the task, the team was given 30 minutes to agree on a final list of eight items from the original list of 25 that they deemed to be the most essential for survival. In addition to the information in the first round, the robot also provided additional facts regarding various environmental factors such as the weather, plants, or geography, for example: "Life threatening temperature is rare, but does occur. Make sure you save up supplies to survive a 3-day long blizzard." This allowed us to provide participants with additional material for further group discussion and encouraged questioning of prior assumptions.

\subsection{Robot Platform and Behaviors}

For our experiment, we used the commercial robot Jibo [12]. Jibo is 11 inches tall and has a 3-axis motor system and a touchscreen face. Jibo responded to the verbal utterances of participants, captured through individual headset microphones and converted from 
Table 1: Examples of the targeted supportive utterances the robot made during the experiment, where [p-name] is a placeholder for the participant's name.

\begin{tabular}{ll}
\hline Targeted Supportive Utterance Example & Type \\
\hline "We need a coffee pot. Good idea [p-name].” & rephrase \\
"Use garbage bag to store the berries. Okay [p-name].” & \\
\hline "Camera. [p-name], I think that's worth considering.” & \multirow{2}{*}{ item } \\
"Soda, chocolate, that makes sense [p-name].” & \\
\hline "Okay, [p-name].” & simple \\
\hline
\end{tabular}

a speech signal to text using Google's speech-to-text API. We programmed Jibo to play the role of a social robot that engages in various forms of supportive social behaviors while providing assistance to players through the supply of valuable task-related information, described in Section 3.2.

In response to participants' speech, we designed the robot to display general social behaviors to establish itself as a present and active member of the human-robot team. In both rounds of the experiment, the robot nodded with a probability of 0.25 in response to detected speech. The robot also responded verbally to detected speech with a probability of 0.15 during both rounds of the experiment: if an item name is detected within the participants' utterance, then with a probability of 0.5 , the robot's verbal response either gave a useful hint about the item (e.g. "Whiskey is a great disinfectant"), or made a general comment using the item(s)'s name (e.g. "Screwdriver, interesting", "Honey, tape, okay"). If no items were detected in the participants' utterance, the robot's verbal response consisted of a generic verbal backchannel (e.g. "Uh huh", "Yeah").

During round two of the experiment, we designed the robot to deliver targeted supportive utterances. These targeted supportive utterances reinforce the ideas of participants and also use the participant's name (robots using participant names has shown importance in building relationships and engaging people $[15,16])$. In the experiment, the robot responded with targeted supportive utterances that either 1) rephrased and supported an idea proposed by a participant (rephrase), 2) supported an item that a participant mentioned (item), or 3) simply showed support to the participant themselves (simple). Examples of the three types of targeted supportive utterances are shown in Table 1. Of the targeted supportive utterances the robot produced during the experiment, $29 \%$ were rephrase, $34 \%$ were item, and $37 \%$ were simple. We programmed the robot to deliver one targeted supportive utterance to each human participant every 4.5 minutes during round two of the experiment. This resulted in participants receiving an average of $5.62(S D=0.86)$ targeted supportive utterances each throughout the course of the experiment.

\subsection{Procedure}

Upon arrival of the participants, an experimenter obtained informed consent and then asked participants to independently fill out a pre-experiment questionnaire. Then, the experimenter informed participants that they would complete a two-part timed activity, to be completed in randomly divided subgroups of sizes one and two, before completing part two as a group of three. The experimenter first set up the single participant (outgroup) in room B. During the approximately 5 minutes the experimenter was setting up the outgroup participant, the participants of the two-member group (the insiders) were given a list of "get to know you" questions in order to further enforce the ingroup-outgroup divide (e.g. "If you didn't sleep, what would you do with your extra time?"), before the experimenter returned to lead the ingroup participants to room A.

In both rooms, the experimenter asked the participants to put on the headsets at their pre-assigned seats. The experimenter then played Jibo's introduction through the tablet, and had each participant practice successfully querying Jibo about one of the survival items. The experimenter then initiated round one of the task on the tablet, which lasted 15 minutes, and left the room.

After the participants finished the first round, the experimenter escorted the outgroup participant to room A to join the ingroup participants. The experimenter then initiated round two of the task on the tablet (which lasted 30 minutes), played Jibo's introduction to the round, and designated one of the participants as the robot liaison using the following language: "In this part, unlike the first, only one of you will be able to ask fibo questions about the items and environment. For all of you this is [participant name]."

After the task finished, the experimenter led the participants outside of the experiment room and administered the post-experiment questionnaire. Finally, participants received a $\$ 10$ cash payment.

\subsection{Measures}

To evaluate how the robot liaison role and the robot's targeted supportive utterances influenced participant inclusion, we analyzed participants' responses to pre- and post-experiment questionnaires, rankings of survival items in both rounds of the experiment, and conversational behavior.

3.5.1 Pre-experiment survey measures. In order to capture preexisting differences between participants, we collected measures of prior familiarity with team members and two personality measures via a survey administered before the human-robot interaction.

Prior familiarity with team members was assessed by asking participants to evaluate their relationships with each of the two participants on a scale from 1 (I have not met this participant before we completed this study together; I do not know them) to 5 (I would consider this participant to be one of my closest friends). Participants were also asked whether they had the phone numbers or social media contact information of their team members.

We also assessed participants' extraversion and emotional intelligence. We measured participants' extraversion because it is a necessary covariate when analyzing data pertaining to the amount of time people spend talking in the group (Section 3.5.3). We measured participants' emotional intelligence because prior work has demonstrated its correlation with team performance [34].

To measure extraversion, we used an abbreviated version of the Revised Eysenck Personality Questionnaire (EPQR-A) [9] that includes six binary ( 0 - no, 1 - yes) response questions such as "Do you tend to keep in the background on social occasions?" to construct a single score between 0 (low extraversion) to 6 (high extraversion). To measure emotional intelligence, we administered the Short Form of the Trait Emotional Intelligence Questionnaire 
(TEIQue-SF) [6], which asks respondents to indicate how much they agree or disagree with a set of 30 statements, such as "I'm usually able to influence the way other people feel", on a 7-point Likert scale from 1 (Strongly Disagree) to 7 (Strongly Agree).

3.5.2 Survival item ranking measures. We examined how similar each of the two subgroup's lists were to the final list of eight items by calculating the absolute difference between the team's final ranking of eight most important items, $r_{\text {fin }}(i)$, from round two, and the ranks initially assigned to these items, $r_{\text {init }}(i)$, by each subgroup in round one, Diff $=\sum_{i=1}^{r_{\text {fin }}} a b s\left(r_{\text {fin }}(i)-r_{\text {init }}(i)\right)$. We normalize the difference scores between the ingroup and outgroup to get our similarity score, e.g. for the outgroup Diffout $=$ Diffout 1 $\left(\right.$ Diff $_{\text {in }}+$ Diff $\left._{\text {out }}\right)$.

We also analyzed how each item on the final list of eight items was initially ranked by each subgroup as either high (ranked 1-8) or low (ranked 9-25), and computed the proportion of those items that made it onto the final list of eight items.

3.5.3 Conversational measures. We investigated several aspects of the conversation that occured between the three participants during the second round of the experiment: each participant's total time spent talking, the standard deviation of the total talking times of each of the three participants in the group, the number of times each item was mentioned, and the proportion of time participants spent talking in response to the robot's targeted supportive utterances.

3.5.4 Post-experiment survey measures. In the post-experiment questionnaire we assessed participants' perceived inclusion by administering the Perceived Group Inclusion Scale (PGIS) [13]. PGIS asks participants to rate agreement with statements like "this group gives me the feeling that I belong" and "this group encourages me to be authentic" on a scale from 1 (Strongly Agree) to 5 (Strongly Disagree). To measure participants' perceptions of Jibo, we used the Robotic Social Attributes Scale (RoSAS) [3]. RoSAS asks respondents to rate how closely they consider descriptor words, each representative of either warmth, competence, or discomfort, to be associated with the robot on a scale from 1 (Definitely Not Associated) to 9 (Definitely Associated).

The post-experiment questionnaire also contained several longresponse questions asking participants to describe the team's interactions on the survival task and the question "Of the two other human participants, which participant would you prefer to work with on a school or work project?” From the responses to this last question, we assigned each participant a preference score. If participant $A$ and participant $C$ specified participant $B$ as their preference, participant B's preference score would be 2 (one for each participant that 'voted' for them). If a participant indicated that they were fine working with both the other participants, each of the other participants received a score increase of 1.0. If a participant said they would prefer working with neither of the other participants, neither of the other participants received any score increase.

\subsection{Participants}

Participants were recruited for this study from a high school program held at Yale University. The students from the program came from 80 different countries, with $46.66 \%$ from the United States. The breakdown by continent is: $52.33 \%$ from North America, $24.27 \%$ from Asia, $11.34 \%$ from Africa, $6.84 \%$ from Europe, $3.20 \%$ from South America, and $2.03 \%$ from Australia.

A total of 30 groups (90 participants) were recruited for participation in this study. Of the 30 groups recruited, 4 groups were excluded due to either not finishing the experimental task or technical difficulties (e.g. a participant's microphone got disconnected disabling them from querying the robot). For the 26 remaining groups (78 participants), 38 participants were female and 40 participants were male. The average age of participants was 16.82 $(S D=0.72)$. There were 6 all female groups, 4 all male groups, 4 groups with 2 females and 1 male, and 12 groups with 1 female and 2 males. For the 16 groups with mixed-gender compositions, we balanced by gender the designation of both the outgroup member ( 9 females, 7 males) and the robot liaison ( 8 females, 8 males). There were 13 groups with an ingroup robot liaison and 13 groups with an outgroup robot liaison.

Participants' familiarity with the other participants in their group $(M=1.10, S D=1.06)$, extraversion $(M=3.90, S D=2.15)$, and emotional intelligence $(M=5.27, S D=0.65)$ were assessed in the pre-experiment questionnaire. Using mutli-level mixed effects models described in Section 4, we did not find any significant differences of these characteristics between either participant designations of robot liaison or participant designations of ingroup-outgroup.

\section{RESULTS}

For our analysis of the participant data, we used linear mixed-effects models in order to account for each participant being in a group of three. We designated intergroup bias (ingroup or outgroup), robot liaison designation (yes or no), the interaction between those two variables, and relevant covariates as fixed effects; and the participant's group as a random effect (random intercept). We tested these models for multicollinearity (variance inflation factor), selected them based on the Bayesian information criterion, and evaluated residual errors for lack of trends and heteroscedasticity. For each fixed effect, the model outputs the linear coefficient $(c)$, the standard error $(S E)$, and the significance $(p)$ value of that predictor.

When analyzing data for each group, we used an analysis of variance (ANOVA) where each group is an independent sample. The main independent variable of interest is whether the robot liaison is an ingroup member or an outgroup member. We used the following covariates in this analysis: the average familiarity of group members and the number of females in the group. The effect size is reported as partial eta squared $\left(\eta^{2}\right)$.

\subsection{Ingroup-Outgroup Differences}

Based on our experimental design that introduced an intergroup bias where one participant (outgroup) completed round one separately from the two other participants (ingroup), we expected that there would be inclusion-related differences between ingroup and outgroup participants. We observed this bias in the similarity of ingroup and outgroup survival item rankings from round one with the final list the team produced after round two, as well as in the post-experiment preferred partner scores.

We analyzed the similarity of the final list of 8 items with both the ingroup and outgroup's initial ranking of those 8 items, where 


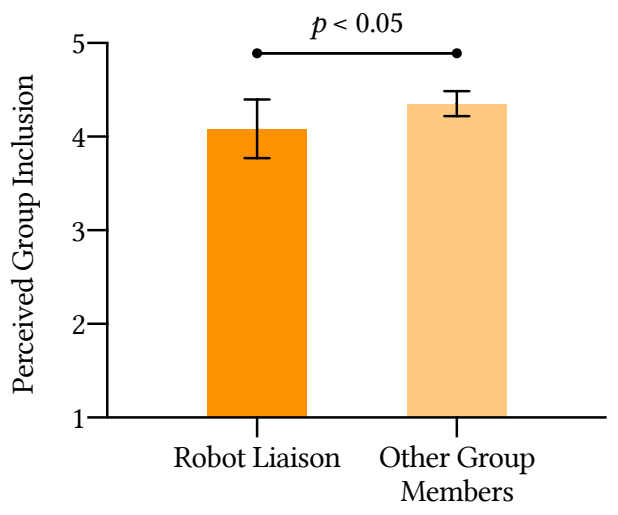

Figure 3: Participants who were the robot liaison had a lower perceived group inclusion than the other group members. Error bars represent a 95\% confidence interval.

smaller values indicate higher similarity of the lists. We used a linear mixed-effects model that best fit the data with emotional intelligence ( $c=-0.05, S E=0.02, p=0.023)$ as a covariate. We found that ingroup members had a more similar ranking of the top 8 items on their initial list $(M=0.45, S D=0.11)$ than outgroup members $(M=0.55, S D=0.11, c=0.10, S E=0.04, p=0.005)$.

We also examined partner preference scores, our measure of how much a participant is preferred as a teammate by their fellow participants. We analyzed the partner preference scores using a linear mixed-effects model that best fit the data, with age $(c=0.21, S E=0.11, p=0.055)$ and emotional intelligence $(c=$ $0.44, S E=0.12, p<0.001)$ as covariates. We discovered that ingroup participants had significantly higher partner preference scores $(M=1.08, S D=0.73)$ than outgroup participants $(M=$ $0.80, S D=0.56, c=-0.51, S E=0.21, p=0.019)$.

These ingroup-outgroup differences support our experimental design of imposing intergroup biases among the three participants. The ingroup's higher similarity between the initial and final item rankings and the higher preference for ingroup members as work partners serve as a manipulation check.

\subsection{Influence of the Robot Liaison Role}

In order to investigate the influence of the robot liaison role on participant inclusion (Research Question 1), we analyzed the perceived group inclusion survey measure as well as the measures of which survival items were included on the team's final list of eight items.

For our analysis of the participants' ratings on the perceived group inclusion scale, the linear mixed-effects model that best fit the data used the covariates of age $(c=-0.20, S E=0.09, p=0.025)$ and maximum familiarity $(c=0.12, S E=0.05, p=0.011)$. We found that participants who were the robot liaison had lower ratings of perceived group inclusion $(M=4.08, S D=0.78)$ than the other group members $(M=4.35, S D=0.49, c=-0.41, S E=0.17, p=$ 0.021), as shown in Figure 3.

In order to analyze the influence of the robot liaison and the ingroup-outgroup designations, we examined the items initially ranked high (items ranked 1-8) and low (items ranked 9-25) by
Table 2: Proportion of survival items that were initially ranked high and low by the ingroup $\left(H_{i n}, L_{i n}\right)$ and outgroup $\left(H_{\text {out }}, L_{\text {out }}\right)$ that made it onto the group's final list of 8 items.
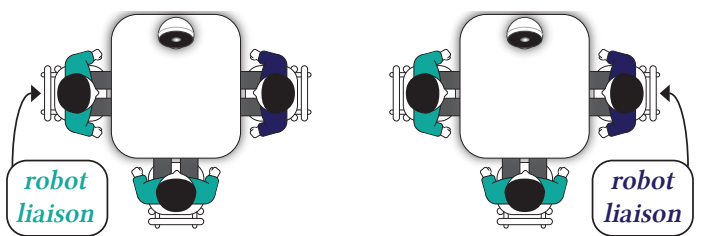

Ingroup Robot Liaison

Outgroup Robot Liaison

\begin{tabular}{lll}
\hline $\boldsymbol{H}_{\text {in }}, \boldsymbol{H}_{\text {out }}$ & 0.71 (2.0/2.8 items) & $0.79(2.6 / 3.2$ items $)$ \\
\hline $\boldsymbol{H}_{\text {in }}, \boldsymbol{L}_{\text {out }}$ & 0.42 (2.1/5.2 items) & $0.40(2.1 / 4.8$ items $)$ \\
\hline $\boldsymbol{L}_{\text {in }}, \boldsymbol{H}_{\text {out }}$ & 0.47 (2.4/5.2 items) & $0.32(1.5 / 4.7$ items $)$ \\
\hline $\boldsymbol{L}_{\text {in }}, \boldsymbol{L}_{\text {out }}$ & 0.14 (1.5/11.8 items) & 0.15 (1.8/12.3 items) \\
\hline
\end{tabular}

each of the 2 subgroups in round one of the experiment. We then calculated the proportion of these items that made it onto the final list of 8 items produced by the entire team at the end of round two of the experiment (see Table 2). We found that a higher proportion of items were chosen that were initially low on the ingroup list and initially high on the outgroup list $\left(L_{i n}, H_{\text {out }}\right)$ if the robot liaison was an ingroup member $(M=0.47, S D=0.14)$ than if the robot liaison was an outgroup member $\left(M=0.32, S D=0.18, F=5.59, \eta^{2}=\right.$ $0.19, p=0.027)$. Thus, when the outgroup member is the robot liaison, as opposed to an ingroup member, the team is less likely to incorporate items favored by the outgroup member.

These findings suggest that the robot liaison role works against efforts to increase inclusion in human team members, resulting in decreased perceived inclusion in the robot liaisons and the incorporation of fewer of the outgroup robot liaison's ideas into the team's final list of items. When asked in the post-experiment questionnaire if the status of any group member influenced the group dynamic, most participants did not think so (e.g. "No, although only one could speak to fibo, we all contributed to the decisions equally"). However a few participants did express a difference in group member status (e.g. "I think that since I was the only one allowed to ask fibo questions it made me more dominant" and "other group members had to ask [participant name] to ask fibo questions"). From these responses, it seems that participants did not overwhelmingly feel a difference in group inclusion or membership because of the robot liaison status. However, some participants did point to a noticeable difference in the power dynamics where the robot liaison was seen as having greater influence than the other members. This could help explain the robot liaison's lower perceived inclusion and, when they were an outgroup member, their reduced likelihood of the group incorporating their ideas.

\subsection{Influence of the Robot's Supportive Utterances}

To investigate the influence of the robot's supportive utterances (Research Question 2), we examined participants' verbal responses to the robot's targeted supportive utterances. In this analysis, we 

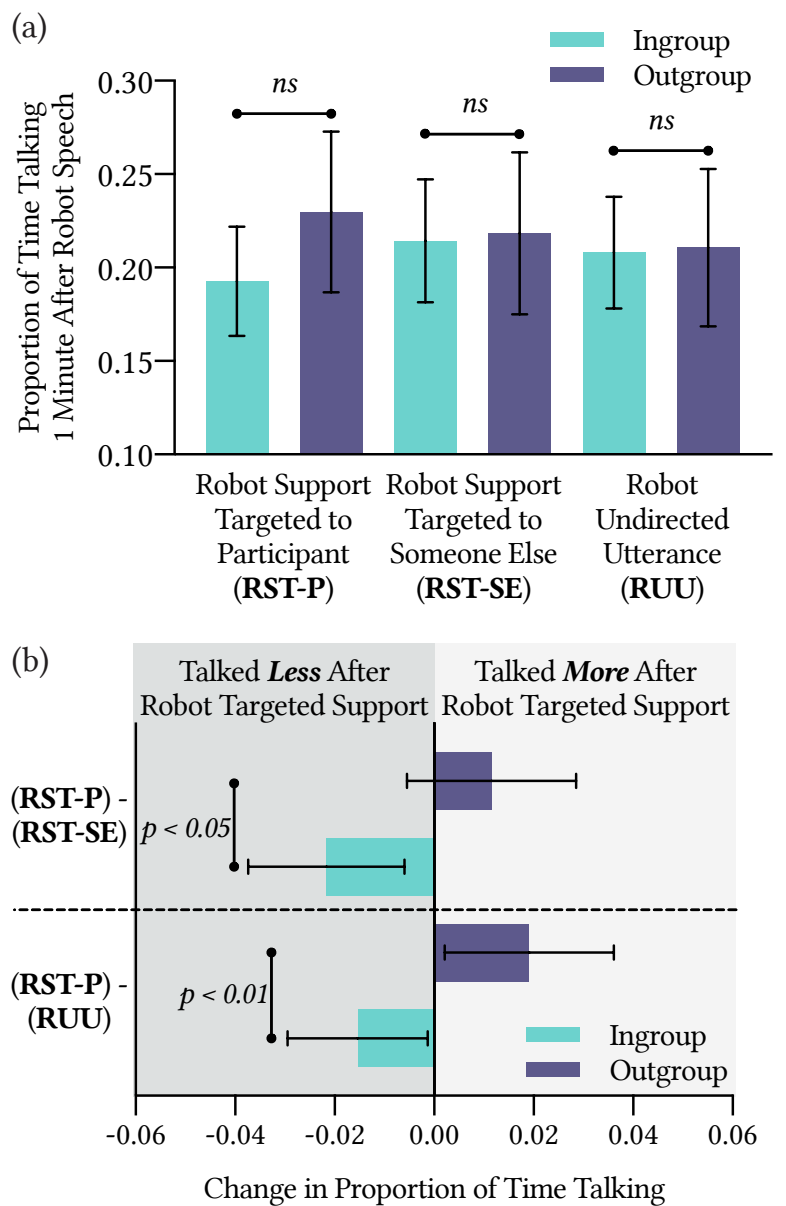

Figure 4: Outgroup participants, as opposed to ingroup participants, displayed a significantly higher difference in the proportion of time they spent talking during the one minute after the robot's support targeted to the participant (RSTP) when compared with two baselines: 1) the proportion of time they spent talking during the one minute after the robot support was targeted to someone else (RST-SE) and 2) the proportion of time they spent talking during the the one minute after a robot undirected utterance (RUU). Error bars represent a $95 \%$ confidence interval.

excluded four participants' data because they did not comply in keeping their microphone on during the experiment. We compared the proportion of time a participant spent talking 1 minute after the robot delivered a targeted supportive utterance (robot support targeted to participant - RST-P) with two controls: 1) the proportion of time a participant spent talking 1 minute after the robot delivered a targeted supportive utterance to someone else (robot support targeted to someone else - RST-SE) and 2) the proportion of time a participant spent talking 1 minute after an undirected utterance from the robot (robot undirected utterance - RUU). As shown in Figure 4(a), ingroup and outgroup participants did not display a significant difference in their proportions of time talking in the 1 minute after robot targeted support to the participant, RST-P, $(c=0.01, S E=0.03, p=0.703)$, in the 1 minute after robot targeted support to someone else, RST-SE, $(c=-0.03, S E=0.03, p=0.445)$, or in the 1 minute after robot undirected utterances, RUU, $(c=$ $-0.03, S E=0.03, p=0.263$ ). These analyses were conducted with linear-mixed effects models that best fit the data with extraversion (RST-P: $c=0.02, S E=0.01, p=0.004$; RST-SE: $c=0.02, S E=$ $0.01, p=0.007$; RUU: $c=0.02, S E=0.01, p=0.002$ ) as a covariate.

We then examined the difference between each participant's proportion of time talking in the 1 minute after robot targeted support to the participant, RST-P, and our two controls (RTS-SE and RUU), Figure 4(b). The linear mixed-effects models that best fit the data for these two analyses did not use any covariates. When examining the difference in participants' talking after the robot gave targeted support to them as opposed to after the robot targeted support to someone else (RST-P - RST-SE), we found that outgroup members $(M=0.012, S D=0.041)$ had a more positive difference than ingroup members $(M=-0.022, S D=0.055, c=0.03, S E=0.02, p=$ $0.047)$, indicating that ougroup members had a higher verbal response to the robot targeted support. In analyzing the difference in participants' talking after robot gave targeted support to them as opposed to after undirected robot utterances (RST-P - RUU), we found that outgroup members $(M=0.019, S D=0.041)$ had a more positive difference than ingroup members $(M=-0.015, S D=$ $0.049, c=0.04, S E=0.02, p=0.007)$ again indicating that ougroup members had a higher verbal response to the robot targeted support. These results suggest that the robot's supportive utterances positively influenced outgroup, but not ingroup, participants' verbal contributions during the task.

In the same analyses described in the prior paragraph, we also found differences between robot liaison participants and the other participants. When examining the difference in participants' talking after the robot gave targeted support to them as opposed to after the robot targeted support to someone else (RST-P - RST-SE), we found that robot liaisons $(M=0.011, S D=0.046)$ had a more positive difference than other group members $(M=-0.022, S D=0.053$, $c=0.03, S E=0.02, p=0.044)$. However, in the difference in participants' talking after robot gave targeted support to them as opposed to after undirected robot utterances (RST-P - RUU), the robot liaison designation had no significant influence $(c=0.02, S E=$ $0.02, p=0.274$ ). Since the increased proportion robot liaison talking time following robot targeted support is only supported by one of our baseline comparisons, it is possible that the robot liaisons have a similar boost in talking in response to robot targeted support as outgroup members, but the effect may not be as pronounced.

\subsection{Perceptions of the Robot}

We evaluated participants' perceptions of the robot by analyzing their responses to the Robotic Social Attributes Scale (RoSAS). Using linear mixed effects models, neither the ingroup-outgroup, robot liaison designations, nor the interaction between the two resulted in significant differences in participants' perceptions of the robot's warmth, competence, or discomfort. Across all participants, the average ratings of the robot's RoSAS attributes, rated on a 1-9 Likert scale, are as follows: warmth $(M=5.81, S D=1.45)$, competence $(M=7.21,1.24)$, and discomfort $(M=2.19, S D=1.10)$. 


\section{DISCUSSION AND CONCLUSION}

In this study, we investigated two different ways in which robot team members can shape inclusion: 1) through a specialized role that gave one participant increased interaction with a robot and 2) through supportive utterances by the robot, targeted to each participant. To investigate the efficacy of these two strategies for increasing inclusion, we designed an experiment in which humanrobot groups with experimentally manipulated ingroup-outgroup divides must work together as teams in a collaborative task.

We were able to confirm that our ingroup-outgroup manipulation was successful. On average, each team's final item rankings were more similar to the initial item rankings made by ingroup members than those made by outgroup members. Moreover, ingroup participants were on average preferred as future work partners over outgroup participants by the other members of the team. Beyond our success in experimentally creating these subgroup divides in this specific study, we contribute to the field of HRI an experimental study design that enables researchers to investigate inclusion and ingroup-outgroup divides in future work.

To explore the influence of a specialized role on team member inclusion, we designated one member of the team the 'robot liaison' that allowed this participant to have privileged communications with the robot. On the one hand, this designation could have promoted team inclusion by providing a sense of value to the team member with the specialized skill. On the other hand, this could have further isolated the member through the addition of another dividing feature with the other members of the group. Our results showed stronger support for this second idea. Participants assigned to the role of the robot liaison reported lower levels of group inclusion than other team members, demonstrating the possible isolating effects of the role. It is possible that the introduction of a robot with exclusive access produces a change in the power dynamics of the team, particularly when the robot plays an essential role. Prior work has established a link between leadership and feelings of isolation and loneliness [26]. Although the robot liaison in this experiment is not explicitly in a leadership position, the increased influence given to the robot liaison could have produced their lower perceived inclusion ratings.

This divide was particularly apparent when the robot liaison was an outgroup member. When an outgroup member was the robot liaison, as opposed to an ingroup member, the group as a whole incorporated fewer survival items favored by the outgroup member. It is likely that giving the robot liaison role to the outgroup member created another divide between the outgroup member and the ingroup members. This increased division may have made the in group members less receptive to the ideas of the outgroup member. This result highlights the dangers of having an already-excluded member of a team take on a specialized role, as the outgroup participants in this study had less of their ideas incorporated into the team's final decision when they were given a specialized role to interact with the robot member of the team.

These findings have important implications for the increasingly prevalent number of human-robot teams today, as robot members are frequently incorporated with a human "liaison." These liaisons may be a specialized robot operator, such as in factory, search and rescue, and surgical teams, or simply a member with more implicit control of the robot, such as team leaders or remote members participating through telepresence. However, as we have shown, this practice can be detrimental to the perceived inclusion of the liaisons themselves, especially if there are already pre-existing faultlines between the liaison and the other members.

In order to investigate our second proposed strategy for increasing team member inclusion, we programmed the robot to make targeted supportive utterances during team discussions. We found that whereas ingroup members appeared to be mostly unaffected by these supportive utterances, outgroup members of the team spoke more in the time immediately after receiving one of the robot's targeted supportive utterances as compared to the times after other utterances made by the robot. Thus, we found evidence for robotemployed targeted supportive utterances improving team inclusion and contribution by providing support and affirmation to relatively excluded team members.

Overall, our results demonstrate that the roles and actions of a robot team member can influence overall team inclusion. We note that two factors, sample representativeness and robot utterance delivery, may have had a non-trivial impact on our results. First, because we recruited participants from a relatively small two week high school program, it is possible that our teams had higher baseline levels of inclusion and common ground, and thus were also less affected by our ingroup-outgroup manipulation. Second, because we implemented relatively simple robot behaviors, the robot would occasionally produce poorly timed or irrelevant utterances (e.g. a robot response of "key, good idea" after a participant had said "I don't think we should bring the key"). Because these two factors may have reduced the effectiveness of our experimental manipulations, either by raising the initial level of team inclusion or by minimizing the beneficial influence of the robot, we believe that the significance of our results in spite of these factors highlights the potential impact of robot team members, and thus the importance of considering the possible consequences of including robot members in teams.

A vast majority of teams consist of members with diverse skill sets, backgrounds, and experience. Inclusion is a critical component to both the success of the team and the commitment of its members $[5,27,29]$. In line with prior work in HRI, we have demonstrated that the social dynamics and behaviors of groups can be shaped by the actions of a robot member. However, we found that these effects are not necessarily always in a positive direction. The results of our experiment show that whereas the actions of robots can be used to promote a sense of team inclusion, the mere presence of robots within a team may produce faultlines and isolate team members if differential abilities to interact with the robot are introduced. As robots are increasingly incorporated into human teams, we recommend that we, as a community, work to better understand and take into account the influence robot members can have on inclusion and other social dynamics of the team, as we seek to promote the success of human-robot teams.

\section{ACKNOWLEDGMENTS}

This work was supported by NSF award IIS-1813651 and ONR award N00014-18-1-2776. We also thank Evelyn Roberts for helping to conduct pilot experiments, and Tom Wallenstein and Sean Hackett for their assistance in data collection. 


\section{REFERENCES}

[1] Minoo Alemi, Ashkan Ghanbarzadeh, Ali Meghdari, and Leila Jafari Moghadam. 2016. Clinical Application of a Humanoid Robot in Pediatric Cancer Interventions. International fournal of Social Robotics 8, 5 (01 Nov 2016), 743-759. https://doi. org/10.1007/s12369-015-0294-y

[2] Andrew Scott Baron and Yarrow Dunham. 2015. Representing âĂŸusâĂŹ and âĂŸthemâĂŹ: Building blocks of intergroup cognition. fournal of Cognition and Development 16, 5 (2015), 780-801.

[3] Colleen M Carpinella, Alisa B Wyman, Michael A Perez, and Steven J Stroessner 2017. The robotic social attributes scale (rosas): Development and validation. In Proceedings of the 2017 ACM/IEEE International Conference on human-robot interaction. ACM, 254-262.

[4] Vijay Chidambaram, Yueh-Hsuan Chiang, and Bilge Mutlu. 2012. Designing persuasive robots: how robots might persuade people using vocal and nonverbal cues. In Proceedings of the seventh annual ACM/IEEE international conference on Human-Robot Interaction. ACM, 293-300.

[5] Sangmi Cho and Michàlle E Mor Barak. 2008. Understanding of diversity and inclusion in a perceived homogeneous culture: A study of organizational commitment and job performance among Korean employees. Administration in Social Work 32, 4 (2008), 100-126.

[6] Andrew Cooper and KV Petrides. 2010. A psychometric analysis of the Trait Emotional Intelligence Questionnaire-Short Form (TEIQue-SF) using item response theory. Fournal of personality assessment 92, 5 (2010), 449-457.

[7] Filipa Correia, Samuel Mascarenhas, Rui Prada, Francisco S. Melo, and Ana Paiva 2018. Group-based Emotions in Teams of Humans and Robots. In Proceedings of the 2018 ACM/IEEE International Conference on Human-Robot Interaction (HRI'18) ACM, New York, NY, USA, 261-269. https://doi.org/10.1145/3171221.3171252

[8] Yarrow Dunham, Andrew Scott Baron, and Susan Carey. 2011. Consequences of âĂIJminimalâĂİ group affiliations in children. Child development 82, 3 (2011), 793-811.

[9] Leslie J Francis, Laurence B Brown, and Ronald Philipchalk. 1992. The development of an abbreviated form of the Revised Eysenck Personality Questionnaire (EPQR-A): Its use among students in England, Canada, the USA and Australia Personality and individual differences 13, 4 (1992), 443-449.

[10] D. Hebesberger, T. Koertner, C. Gisinger, J. Pripfl, and C. Dondrup. 2016. Lessons learned from the deployment of a long-term autonomous robot as companion in physical therapy for older adults with dementia a mixed methods study. In 2016 11th ACM/IEEE International Conference on Human-Robot Interaction (HRI) 27-34. https://doi.org/10.1109/HRI.2016.7451730

[11] Guido Hertel and Joachim Hüffmeier. 2011. Many cheers make light the work: How social support triggers process gains in teams. Fournal of Managerial Psychology (2011).

[12] Jibo Inc. [n.d.]. Jibo. https://jibo.com

[13] Wiebren S Jansen, Sabine Otten, Karen I van der Zee, and Lise Jans. 2014. Inclusion Conceptualization and measurement. European journal of social psychology 44, 4 (2014), 370-385.

[14] Malte F. Jung, Nikolas Martelaro, and Pamela J. Hinds. 2015. Using Robots to Moderate Team Conflict: The Case of Repairing Violations. In Proceedings of the Tenth Annual ACM/IEEE International Conference on Human-Robot Interaction (HRI '15). ACM, New York, NY, USA, 229-236. https://doi.org/10.1145/2696454.2696460

[15] Takayuki Kanda, Takayuki Hirano, Daniel Eaton, and Hiroshi Ishiguro. 2004 Interactive robots as social partners and peer tutors for children: A field trial. Human-Computer Interaction 19, 1-2 (2004), 61-84.

[16] Takayuki Kanda, Rumi Sato, Naoki Saiwaki, and Hiroshi Ishiguro. 2007. A twomonth field trial in an elementary school for long-term human-robot interaction. IEEE Transactions on robotics 23, 5 (2007), 962-971.

[17] Cory D Kidd and Cynthia Breazeal. 2004. Effect of a robot on user perceptions. In 2004 IEEE/RSf International Conference on Intelligent Robots and Systems (IROS)(IEEE Cat. No. 04CH37566), Vol. 4. IEEE, 3559-3564.

[18] J Clayton Lafferty and Alonzo W Pond. 1974. The desert survival situation: A group decision making experience for examining and increasing individual and team effectiveness. Human Synergistics.

[19] Dora C Lau and J Keith Murnighan. 1998. Demographic diversity and faultlines: The compositional dynamics of organizational groups. Academy of Management Review 23, 2 (1998), 325-340.

[20] Jin Joo Lee, Fei Sha, and Cynthia Breazeal. 2019. A Bayesian Theory of Mind Approach to Nonverbal Communication. In 2019 14th ACM/IEEE International
Conference on Human-Robot Interaction (HRI). IEEE, 487-496.

[21] Iolanda Leite, André Pereira, Samuel Mascarenhas, Carlos Martinho, Rui Prada, and Ana Paiva. 2012. The influence of empathy in human-robot relations. International Fournal of Human-Computer Studies 71 (01 2012). https://doi.org/10. 1016/j.ijhcs.2012.09.005

[22] Frederick A Miller. 1998. Strategic culture change: The door to achieving high performance and inclusion. Public personnel management 27, 2 (1998), 151-160.

[23] Raquel Oliveira, Patrícia Arriaga, Patrícia Alves-Oliveira, Filipa Correia, Sofia Petisca, and Ana Paiva. 2018. Friends or Foes?: Socioemotional Support and Gaze Behaviors in Mixed Groups of Humans and Robots. In Proceedings of the 2018 ACM/IEEE International Conference on Human-Robot Interaction (HRI '18). ACM, New York, NY, USA, 279-288. https://doi.org/10.1145/3171221.3171272

[24] Cliff Oswick and Mike Noon. 2014. Discourses of diversity, equality and inclusion: trenchant formulations or transient fashions? British fournal of Management 25, 1 (2014), 23-39.

[25] André Pereira, Iolanda Leite, Samuel Mascarenhas, Carlos Martinho, and Ana Paiva. 2011. Using Empathy to Improve Human-Robot Relationships. In HumanRobot Personal Relationships, Maarten H. Lamers and Fons J. Verbeek (Eds.). Springer Berlin Heidelberg, Berlin, Heidelberg, 130-138.

[26] Ami Rokach. 2014. Leadership and loneliness. International Journal of Leadership and Change 2, 1 (2014), 6.

[27] Meghna Sabharwal. 2014. Is diversity management sufficient? Organizational inclusion to further performance. Public Personnel Management 43, 2 (2014), 197-217.

[28] Solace Shen, Petr Slovak, and Malte F. Jung. 2018. "Stop. I See a Conflict Happening.": A Robot Mediator for Young Children's Interpersonal Conflict Resolution. In Proceedings of the 2018 ACM/IEEE International Conference on Human-Robot Interaction (HRI '18). ACM, New York, NY, USA, 69-77. https: //doi.org/10.1145/3171221.3171248

[29] Lynn M Shore, Amy E Randel, Beth G Chung, Michelle A Dean, Karen Holcombe Ehrhart, and Gangaram Singh. 2011. Inclusion and diversity in work groups: A review and model for future research. Journal of management 37,4 (2011), 1262-1289.

[30] Elaine Short and Maja J. Matarić. 2017. Robot moderation of a collaborative game: Towards socially assistive robotics in group interactions. In 26th IEEE International Symposium on Robot and Human Interactive Communication (RO-MAN '17). IEEE Press, Lisbon, Portugal, 385-390. https://doi.org/10.1109/ROMAN.2017.8172331

[31] Sarah Strohkorb, Ethan Fukuto, Natalie Warren, Charles Taylor, Bobby Berry, and Brian Scassellati. 2016. Improving human-human collaboration between children with a social robot. In 25th IEEE International Symposium on Robot and Human Interactive Communication (RO-MAN '16). IEEE Press, New York, NY, USA, 551-556. https://doi.org/10.1109/ROMAN.2016.7745172

[32] Sarah Strohkorb Sebo, Margaret Traeger, Malte Jung, and Brian Scassellati. 2018. The Ripple Effects of Vulnerability: The Effects of a Robot's Vulnerable Behavior on Trust in Human-Robot Teams. In Proceedings of the 2018 ACM/IEEE International Conference on Human-Robot Interaction (HRI '18). ACM, New York, NY, USA, 178-186. https://doi.org/10.1145/3171221.3171275

[33] Maria Stubbe. 1998. Are you listening? Cultural influences on the use of supportive verbal feedback in conversation. Journal of Pragmatics 29, 3 (1998), 257-289.

[34] Elizabeth Stubbs Koman and Steven B Wolff. 2008. Emotional intelligence competencies in the team and team leader: A multi-level examination of the impact of emotional intelligence on team performance. fournal of Management Development 27, 1 (2008), 55-75.

[35] Henri Tajfel. 1982. Social psychology of intergroup relations. Annual review of psychology 33, 1 (1982), 1-39.

[36] Hamish Tennent, Solace Shen, and Malte Jung. 2019. Micbot: A Peripheral Robotic Object to Shape Conversational Dynamics and Team Performance. In 14th ACM/IEEE International Conference on Human-Robot Interaction (HRI '19). IEEE Press, Daegu, South Korea, 133-142. https://doi.org/10.1109/HRI.2019.8673013

[37] Dina Utami and Timothy Bickmore. 2019. Collaborative User Responses in Multiparty Interaction with a Couples Counselor Robot. In 14th ACM/IEEE International Conference on Human-Robot Interaction (HRI '19). IEEE Press, Daegu, South Korea, 294-303. https://doi.org/10.1109/HRI.2019.8673177

[38] Nigel Ward and Wataru Tsukahara. 2000. Prosodic features which cue backchannel responses in English and Japanese. Journal of pragmatics 32, 8 (2000), 1177-1207. 